\title{
Balloon-Catheter Buddy Technique for Coiling of Very Small Aneurysm
}

\author{
Vikas Bhatia ${ }^{1}$ Bharat Hosur ${ }^{1}$ Ajay Kumar ${ }^{1}$ \\ 1Department of Radiodiagnosis, Post Graduate Institute of Medical \\ Education and Research, Chandigarh, India
}

J Clin Interv Radiol ISVIR 2021;6:68-70.

\begin{abstract}
Address for correspondence Vikas Bhatia, DM, Neuroradiology Section, Department of Radiodiagnosis, Post Graduate Institute of Medical Education and Research,Chandigarh 160012, India (e-mail: drvikasbhatia@gmail.com).
\end{abstract}
Abstract Keywords
- coiling
- very small aneurysms
- catheter

The catheter movement and stability in coiling of very small aneurysms is challenging. We describe a technique for controlled catheter movement and successful coiling of a very small aneurysm.

\section{Introduction}

Management of very small $(<3 \mathrm{~mm})$ aneurysms through the endovascular route is challenging, especially in presence of rupture and unfavorable anatomy. Due to their small size, it is very important to have accurate microcatheter positioning and coil placement to prevent complications such as rupture and coil prolapse. We describe our technique in such a challenging case.

\section{Case Report}

A 35-year-old man presented to our institute with a sudden-onset headache for the last 4 days. His noncontrast computed tomography (NCCT) head revealed modified Fischer grade III subarachnoid hemorrhage. His clinical examination revealed E4V5M6 status, World Federation of Neurosurgeons (WFNS) grade I, and Hunt and Hess grade II. He had a history of drug abuse for the past 5 years. A computed tomography (CT) angiography head was done which revealed a very small outpouching arising from left A1 anterior cerebral artery (ACA). He was taken for diagnostic digital subtraction angiography (DSA) which showed the presence of a very small aneurysm of size $2.6 \mathrm{~mm} \times 2.2 \mathrm{~mm}$ with the neck of $1.5 \mathrm{~mm}$ arising from proximal left A1 ACA which was directed posterosuperior. There was severe vasospasm involving the anterior circulation. The patient was taken for endovascular coiling.

published online June 29, 2021
DOI https://doi.org/

$10.1055 / \mathrm{s}-0041-1729779$

ISSN 2457-0214
The Traxcess (MicroVention, Tustin, California) microwire and Echelon-10 microcatheter (Medtronic, Minneapolis, Minnesota) were advanced into the left A2 ACA. Repeated attempts to cannulate the aneurysm with gentle withdrawal of microcatheter from distal ACA or careful advancement over the wire into the sac met with failure as there was a tendency of microcatheter to fall into the left middle cerebral artery (MCA). Repeat shaping of wire and microcatheter also failed to cannulate the aneurysmal sac. There were multiple challenges with this aneurysm such as very small size, location at proximal A1 ACA close to internal carotid artery (ICA) bifurcation, steep angulation of A1 ACA, and posterosuperior direction of the aneurysm. With these challenges, the controlled fine movement of the microcatheter was not possible and it was repeatedly falling into the left MCA.

We employed a different technique ( $\boldsymbol{- \text { Fig. }} \mathbf{1}$ ) to successfully place the microcatheter at the aneurysm neck. The first step was to place the balloon catheter (Sceptor XC $4 \mathrm{~mm} \times$ $11 \mathrm{~mm}$, MicroVention Inc, Tustin, California) into the left M1 MCA over the microwire (-Fig. 2). The microwire and microcatheter were then advanced into left A2 ACA. The proximity of the microcatheter and balloon catheter as buddy allowed the natural friction resulting in controlled movement of the coiling microcatheter and it was successfully placed near the neck of the aneurysm. The buddy technique allowed for stable positioning of the coiling microcatheter and a $2 \mathrm{~mm} \times 2 \mathrm{~mm}$ Axium prime extra soft (ev3, Irvine, CA,

(c) 2021. Indian Society of Vascular and Interventional Radiology.

This is an open access article published by Thieme under the terms of the Creative Commons Attribution-NonDerivative-NonCommercial-License, permitting copying and reproduction so long as the original work is given appropriate credit. Contents may not be used for commercial purposes, or adapted, remixed, transformed or built upon. (https://creativecommons.org/licenses/by-nc-nd/4.0/).

Thieme Medical and Scientific Publishers Pvt. Ltd. A-12, 2nd Floor, Sector 2, Noida-201301 UP, India 

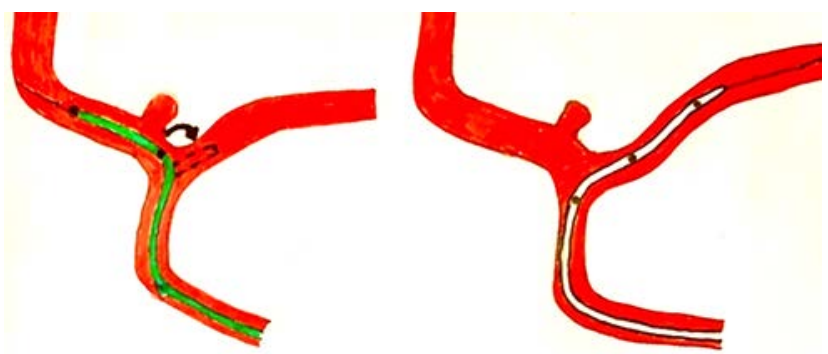

A

b
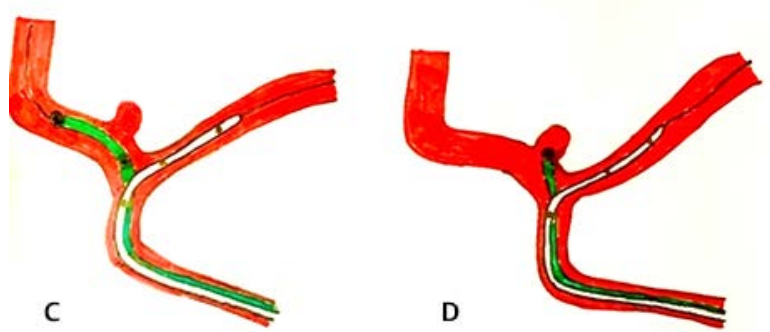

Fig. 1 (A) Maneuvering of the shaped coiling catheter resulted in repeated fall into the left middle cerebral artery (MCA) (curved arrow, dashed catheter) due to sharp angulation of left A1 anterior cerebral artery (ACA) and the proximity of a very small aneurysm to the ICA bifurcation. (B) Placement of balloon in the left MCA. (C) Advancement of coiling microcatheter into the left A2 ACA. (D) Buddy effect of the microcatheter and balloon resulted in a controlled movement and secure placement of the microcatheter tip into the neck of the aneurysm.

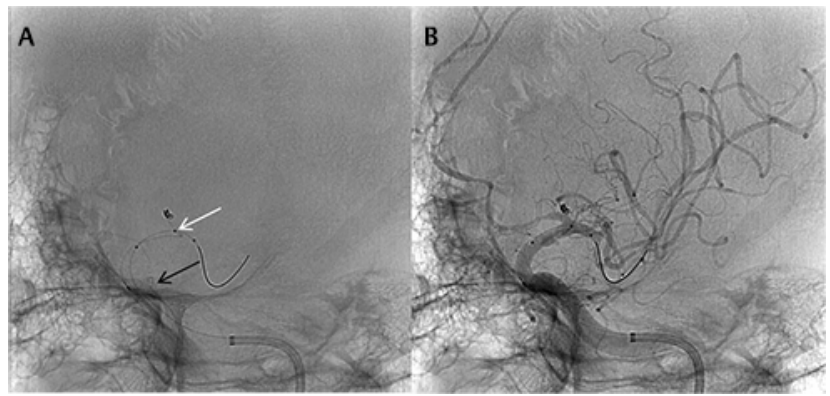

Fig. 3 (A) Digital subtraction angiography (DSA) oblique working position run shows the stable placement of the microcatheter at the aneurysm neck with buddy support from the balloon (white arrow) even though there were considerable tension and looping of microcatheter in proximal ICA(black arrow). (B) Successful placement of small coil in the aneurysm with complete obliteration and normal opacification of anterior cerebral artery (ACA) and middle cerebral artery (MCA) vessels.

USA) coil was placed successfully ( - Fig. 3 ). Complete nonopacification of the aneurysm was achieved and the coil was detached after 10 minutes. However, during microcatheter retrieval, a small loop migrated into the left A1 ACA ( - Fig. 4). Repeated angiographic runs over 30 minutes showed stability of coil mass, nonopacification of the aneurysm, and normal opacification of left ACA. The patient was extubated and had no focal deficit. After two days, the patient developed right hemiparesis with grade III power. Repeat angiography showed stable coil mass with nonopacification of the aneurysm and patent left A1 ACA. There was severe vasospasm in

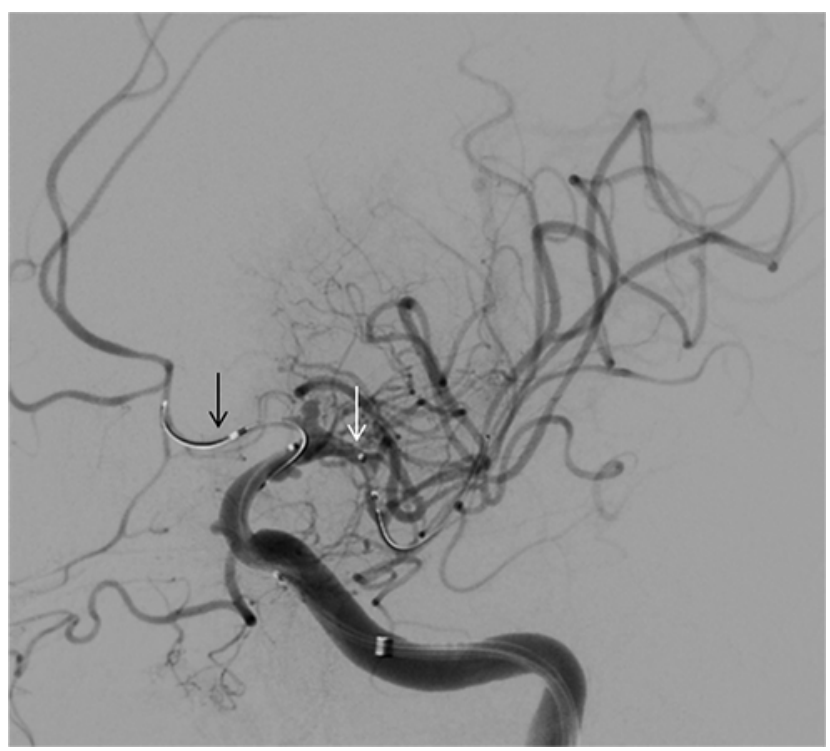

Fig. 2 Digital subtraction angiography (DSA) oblique working position run shows the placement of microcatheter (black arrow) in the left $\mathrm{A} 2$ anterior cerebral artery (ACA) and balloon (right arrow) in left M1 middle cerebral artery (MCA).

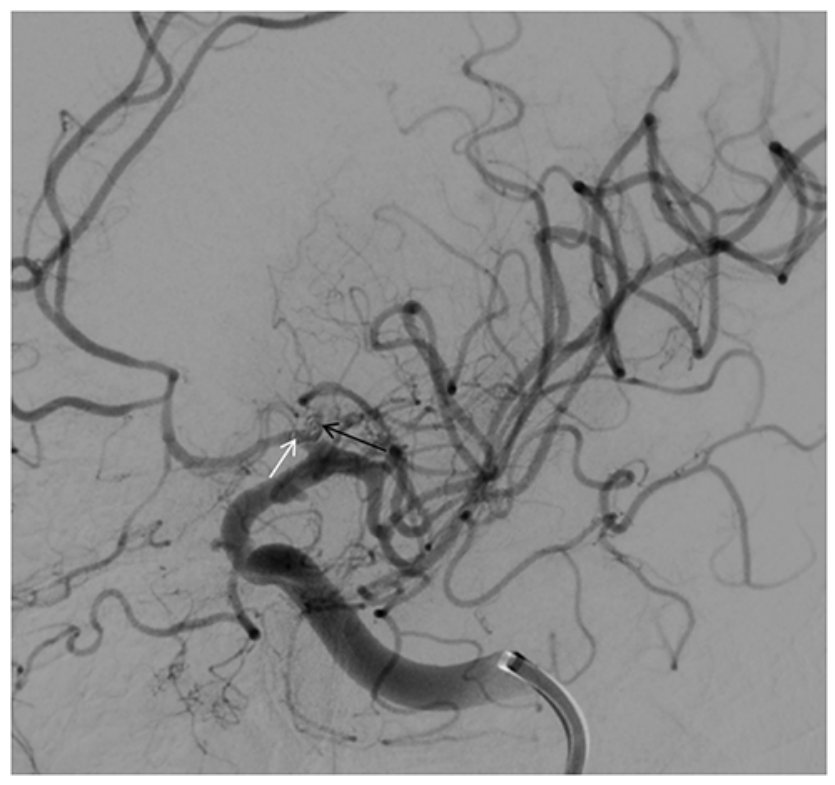

Fig. 4 Digital subtraction angiography (DSA) oblique working position run shows the migration of small coil loop in the left $A 1$ anterior cerebral artery (ACA) (white arrow) with normal opacification of the ACA. The aneurysm shows complete obliteration (black arrow).

the left MCA/ACA territory for which intra-arterial nimodipine was given. He was started on intravenous (IV) milrinone for the next 48 hours which resulted in some improvement in power. No infarcts were seen on the NCCT head. After 1 week he was discharged with E4 V5 M6 status and mild residual right hemiparesis.

\section{Discussion}

While very small unruptured aneurysms can be followed based on their imaging characteristics, the rupture aneurysms need urgent treatment. ${ }^{1}$ Surgical treatment of very 
small aneurysms is challenging due to their small size and thin walls rendering them difficult for clipping as the risk of vessel injury is high. ${ }^{2}$

Similarly, small size carries high risk with endovascular option due to potential chance of perforation by microcatheter and difficult coil placement. ${ }^{3}$

The use of stent or flow diverter in a ruptured aneurysm and small-caliber vessel carries higher bleeding risk due to associated antiplatelet therapy requirement. The use of stent or balloon assistance for very small aneurysms was found to have increased periprocedural complications. ${ }^{4}$ Simple endovascular coiling is a time-tested treatment modality for the obliteration of an aneurysm sac and if performed successfully can prevent the thromboembolic and hemorrhagic complications associated with the use of a balloon or stent. ${ }^{5}$

The greatest challenge with endovascular coiling in a very small aneurysm is limited operator freedom in microcatheter movement particularly in challenging anatomy. The uncontrolled to and fro movement of the microcatheter can result in perforation, repeated fall of the catheter out of the sac into the vessel, or inadvertent positioning of catheter tip near the rupture site of the aneurysm. ${ }^{6}$ Thus, it is of paramount importance to have a controlled movement of the microcatheter so that its tip can be securely placed near the neck.

We used the balloon-catheter buddy technique in our case, to coil the very small aneurysm in the proximal A1 ACA. There were two advantages of placing the balloon in the left MCA across the ICA bifurcation. First, the buddy effect of the balloon provided the required friction for controlled movement of microcatheter rather than random unpredictable to and fro motion due to arterial pulsatility. This resulted in careful stable positioning of the microcatheter tip at the neck of the aneurysm. Second, the balloon across the bifurcation provided the proximal control if required for inadvertent rupture. We did not inflate the balloon during the procedure while deploying the coils as the catheter was stable due to the buddy effect. However, if required, partial inflation can be done to provide stability to the coiling microcatheter. The balloon was not placed into the left A1 ACA due to the small caliber of the vessel and high risk of thromboembolism. Previous studies have also cautioned with the use of a balloon, and have suggested careful intermittent and partial inflation, if required. ${ }^{4,5}$

Previously, few techniques have been described for very small aneurysm catheter placement. ${ }^{6} \mathrm{~A}$ "wireless" technique utilizes the initial placement of the microcatheter beyond the aneurysm, followed by gentle withdrawal till the neck of the aneurysm. The microwire is kept just inside the catheter and gentle spinning is done to point the microcatheter toward the aneurysm. This technique is, however, difficult to employ in the anterior circulation and when the proximal loops are tortuous. ${ }^{6}$

A headhunter shape of microcatheter to engage both the aneurysm and taking support from the opposite wall of the parent vessel has also been described to successfully cannulate the aneurysm. ${ }^{6}$ This technique is better suited for sidewall aneurysms in proximal large vessels. In our case, due to the location of the aneurysm, shaping alone could not provide stable access.

Another challenge in such cases is to deploy a small coil carefully. The literature on very small aneurysm coiling suggests placing a small size soft coil without excessive manipulation. ${ }^{4,5}$ Studies have shown the utility of placing a single appropriate-sized coil, accepting the under coiling, and ignoring any small loop herniating outside the sac. ${ }^{4}$ In our case, we specifically used a soft coil with mechanical detachment. We purposefully used this coil as our plan included placement of a single coil and then waiting for a while to see for the stability of the coil after deployment. This coil by having mechanical detachment had fewer chances of failure to detach after prolonged deployment.

We were able to carefully place the coil inside the sac with stable microcatheter positioning. The coil mass remained stable for the next 10 minutes. However, during retrieval of the microcatheter, there was a migration of a small loop into the left A1 ACA. The loop remained stable and the aneurysm was completely obliterated. After observing for 30 minutes, final check runs were taken and the patient was extubated.

A previous study has also shown acceptance of small migrated coil to be safe with good clinical outcome in very small aneurysms. ${ }^{4}$

We describe this balloon-catheter buddy technique for controlled microcatheter movement and adequate placement of microcatheter tip near the neck of a very small aneurysm. Any uncontrolled movement of microcatheter can have catastrophic complications; we believe that this technique can give control to the operating interventionist for careful maneuvering in very small aneurysm with the added advantage of the presence of a balloon in case of inadvertent rupture.

\section{Conflict of Interest}

None declared.

\section{References}

1 Wiebers DO, Whisnant JP, Huston J III, et al. International Study of Unruptured Intracranial Aneurysms Investigators. Unruptured intracranial aneurysms: natural history, clinical outcome, and risks of surgical and endovascular treatment. Lancet 2003;362(9378):103-110

2 Fujiwara S, Fujii K, Nishio S, Fukui M. Long-term results of wrapping of intracranial ruptured aneurysms. Acta Neurochir (Wien) 1990;103(1-2):27-29

3 Nguyen TN, Raymond J, Guilbert F, et al. Association of endovascular therapy of very small ruptured aneurysms with higher rates of procedure-related rupture. J Neurosurg 2008;108(6):1088-1092

4 Starke RM, Chalouhi N, Ali MS, Penn DL, et al. Endovascular treatment of very small ruptured intracranial aneurysms: complications, occlusion rates and prediction of outcome. J Neurointerv Surg 2013;5(Suppl 3):66-71

5 Gupta V, Chugh M, Jha AN, Walia BS, Vaishya S. Coil embolization of very small ( $2 \mathrm{~mm}$ or smaller) berry aneurysms: feasibility and technical issues. AJNR Am J Neuroradiol 2009;30(2):308-314

6 Dalfino J, Nair AK, Drazin D, Gifford E, Moores N, Boulos AS. Strategies and outcomes for coiling very small aneurysms. World Neurosurg 2014;81(5-6):765-772 